\title{
Molecular Targeting of Dermatofibrosarcoma Protuberans: A New Approach to a Surgical Disease
}

Grant A. McArthur, PhD, East Melbourne and Fitzroy, Australia

Key Words

Dermatofibrosarcoma protuberans, surgery, platelet-derived growth factor receptor, radiotherapy, imatinib, skin sarcoma

\begin{abstract}
Dermatofibrosarcoma protuberans (DFSP) is a low-grade malignancy of the skin and subcutaneous tissues that only rarely forms distant metastases. More than $90 \%$ of cases are associated with a chromosomal translocation involving the COL1A1 gene on chromosome 17 and the platelet-derived growth factor B gene on chromosome 22. Management of this disease is primarily surgical with excellent rates of local control obtained using either wide local excision or Mohs micrographic surgery. Data have recently shown that inhibiting platelet-derived growth factor receptors (PDGFR) with imatinib can induce high rates of clinical response in patients with unresectable or metastatic DFSP. These data have led to approval of imatinib by the U.S. Food and Drug Administration for treating uresectable DFSP. Although wide surgical excision remains standard care, patients with locally advanced disease not suitable for surgical excision can be treated with the PDGFR-inhibitor imatinib, which sometimes allows residual DFSP to be surgically excised. (JNCCN 2007;5:557-562)
\end{abstract}

Dermatofibrosarcoma protuberans (DFSP) is a rare lowgrade malignancy involving the skin and subcutaneous tissues. Unlike other soft tissue sarcomas, it rarely forms distant metastases ( $<5 \%$ of cases) and the clinical challenge in managing this disease arises from its pattern of infiltrative growth that leads to local recurrence after

From the Peter MacCallum Cancer Centre, East Melbourne, Australia and the Department of Medicine, St. Vincent's Hospital, Fitzroy, Australia.

Submitted January 4, 2007; accepted for publication February 6, 2007. Dr. McArthur has been a consultant for and received research support from Novartis and Pfizer, and has received honoraria from Novartis. Dr. McArthur is also supported by a Weary Dunlop Clinical Research Fellowship of the Cancer Council of Victoria.

Correspondence: Grant McArthur, MB, BS, PhD, Peter MacCallum Cancer Centre, St. Andrews Place, East Melbourne, Australia 3002.

E-mail: grant.mcarthur@petermac.org definitive surgical attempts. Recent progress has identified the underlying molecular basis of the disease and has led to the development of targeted therapies for DFSP. This article examines recent evidence for optimal management of DFSP, including the use of imatinib mesylate, which is now approved by the U.S. Food and Drug Administration (FDA) for managing "adult patients with unresectable, recurrent and/or metastatic dermatofibrosarcoma protuberans (DFSP).”

\section{Presentation and Diagnosis of DFSP}

DFSP commonly presents in younger adults with a cutaneous or subcutaneous mass that usually has been present for many years with gradual growth., ${ }^{2,3}$ Frequently, the diagnosis is not suspected until biopsy shows the classical morphology of a fibroblastic tumor with a storiform pattern of cell alignment. DFSP most commonly occurs on the trunk $(35 \%-75 \%),{ }^{2-4}$ where wide margins of surgical resection are usually easily obtained, but also occurs on the limbs and in the head and neck region, often requiring potentially morbid reconstructive surgery. ${ }^{3}$

DFSP is diagnosed based on morphology showing spindle cells that form swirling or storiform patterns. The mitotic count is usually low. Immunohistochemistry is useful because more than $80 \%$ of DFSPs are CD34+. Immunohistochemistry also helps differentiate these tumors from dermatofibromas, fibrosarcomas, or malignant fibrous histiocytomas. ${ }^{7,8}$ Dermatofibromas are typically negative for CD34 and positive for FXIIIa, stromelysin 3, and tenascin. ${ }^{69}$ One significant histologic variant of the disease is DFSP with fibrosarcomatous elements (FSDFSP). This variant demonstrates higher grade histology with nuclear pleomorphism, the presence of mitotic figures and increased cellularity. FS-DFSPs have a more 
aggressive course with an elevated risk for local recurrence $(14 \%-52 \%)$ and distant metastases (8\%-29\%) compared with DFSP without fibrosarcomatous elements, which has risks of approximately $3 \%$ to $16 \%$ and less than $2 \%$ for local recurrence and distant metastases, respectively. ${ }^{2,310}$ Therefore, a synoptic style of pathology reporting is useful, containing the following elements: microscopic description of the tumor; size; microscopic surgical margins; the presence of fibrosarcomatous elements; mitotic count; results of a CD34 stain; and, in certain cases, molecular analyses or cytogenetics. These parameters allow managing clinicians to be confident of the diagnosis and provide some indication of the risk for recurrence, which is useful in planning follow-up.

\section{Molecular Biology of DFSP}

The identification of a chromosomal translocation $\mathrm{t}(17 ; 22)(\mathrm{q} 22 ; \mathrm{q} 13)$ in more than $90 \%$ of DFSP has led directly to the application of targeted therapy. ${ }^{11,12}$ The $t(17 ; 22)$ results in fusion of the COL1A1 and PDGFB genes that encodes a fusion transcript, which then encodes a fusion protein that is processed into fully functional platelet-derived growth factor B (PDGFB). ${ }^{13}$ Because PDGFB is a ligand for both PDGF receptor (PDGFR) $\beta$ and PDGFR $\alpha$, pharmacologic inhibition of these active receptors has been investigated for the treatment of DFSP. The $t(17 ; 22)$ translocation is frequently associated with a supernumerary ring chromosome that contains increased copy numbers of COL1A1 and PDGFB genes and is associated with a characteristic gene-expression profile. ${ }^{14,15}$ Alternative chromosome fusions also have also been described, including $\mathrm{t}(9 ; 22)(\mathrm{q} 32 ; \mathrm{q} 12.2)$ and other more complex karyotypes. ${ }^{14,16-18}$ These more complex genetic changes usually involve chromosome 22 , which contains the PDGFB gene, and therefore experts believe that these genomic abnormalities result in transcripts that may encode functional PDGFB. ${ }^{14,19}$ These observations suggest that that dysregulated expression of PDGFB is the critical molecular event in the pathogenesis of DFSP. Because some genomic changes involving chromosome 22 are only detected with in situ hybridization or comparative genomic hybridisation, simple karyotyping cannot be relied on to detect changes in the PDGFB gene. ${ }^{14}$

Although histology and immunohistochemistry for CD34 are reliable in diagnosing DFSP, karyotyp- ing or in situ hybridization techniques to detect $\mathrm{t}(17 ; 22)$ can be useful in cases posing diagnostic difficulties. $^{14}$

\section{Surgical Management of DFSP}

The standard treatment of DFSP is wide surgical excision. The surgical goal is to achieve clear margins, although the ideal width of the surgical margins remains uncertain. Standard practice is to use margins typical for excision of soft tissue sarcomas, with 2 to $4 \mathrm{~cm}$ often believed ideal., ${ }^{40,21}$ These margins are significantly greater than those recommended for managing other malignant skin tumors, such as melanoma or non-melanoma skin cancer. ${ }^{22,23}$

Some investigators advocate using Mohs micrographic surgery for resecting DFSP. The Mohs technique uses intraoperative assessment of resection margins to detect microscopic tumor cells to help decide the extent of surgery. However, this technique is not widely used internationally, and partly because of resource issues related to surgical time and pathologic examination. ${ }^{24}$ Clinical outcomes from Mohs micrographic surgical technique have been very good, with low reported rates of local recurrence ranging from $0 \%$ to $7 \%$ in series with follow-up of more than 40 months. ${ }^{25-27}$ However, a single institution has shown excellent outcomes using wide margins without Mohs technique. ${ }^{3}$ Similar results have also been obtained with wide excision in other single centers with expertise in sarcoma and skin surgery. ${ }^{28}$ Mohs technique has not been evaluated in randomized trials for any indication, ${ }^{24}$ and opinions continue to differ about its usefulness.

In the series by Fiore et al., 66 of 218 patients treated with wide excision required reconstructive surgery. These investigators also examined the outcomes of patients undergoing surgery for recurrent disease, a frequent problem in DFSP reported in approximately $25 \%$ of cases. ${ }^{2}$ Disease control was obtained with surgery alone in $95 \%$ of patients with recurrent disease, ${ }^{3}$ indicating that for most of these patients, molecular targeted therapies are not first-line treatment.

Given the slow rate of progression of DFSP, follow-up routinely involves regular physical examination at 6 - to 12 -month intervals. ${ }^{29}$ Few data are available to guide the use of imaging in follow-up of DFSP. Because of the low rate of distant metastases, 
regular computed tomography (CT) scanning, which is used in some patients with other soft tissue sarcomas, is difficult to justify. ${ }^{30}$ Ultrasonography and magnetic resonance imaging (MRI) can also be useful in preoperatively assessing the extent of local disease and monitoring for local recurrence. ${ }^{31,32}$

\section{Treatment of Unresectable DFSP: Targeted Therapy or Radiotherapy?}

DFSP that is completely unresectable is a rare occurrence, with only 2 of 159 patients in the series from Bowne et al. ${ }^{2}$ unable to undergo complete resection. However, surgeons and oncologists more frequently encounter difficult situations in which aggressive surgical resection may be accompanied by significant functional or cosmetic morbidity, and therefore nonsurgical means are used to control and palliate the disease. These patients currently have 3 options: 1) treatment on clinical trials, 2) radiotherapy, or 3) targeted therapy with imatinib. In principle, enrollment in a clinical trial should always be considered after treatment failure or for patients who are not surgically curable. However, access to appropriate clinical trials is often difficult for rare malignancies such as DFSP, and therefore radiotherapy or targeted therapy with imatinib is a current option for many patients. Experts have limited experience with radiotherapy in patients with unresectable DFSP. Suit et al. ${ }^{33}$ reported disease control in 3 of 3 patients treated with 67 to 75 Gy of external electron bean radiotherapy coupled with brachytherapy. They treated another 3 patients with preoperative radiotherapy and were able to control disease in 2. Ballo et al. ${ }^{34}$ treated 2 patients with preoperative radiotherapy, and 1 patient with bulky aggressive disease experienced treatment failure. Fortunately recurrence in a previous radiation field is very uncommon and often difficult to manage surgically. Molecularly targeted therapy would be one option for these patients (later discussion). Therefore, one can conclude that radiotherapy is effective in treating DFSP, but its role in patients with unresectable disease is unclear compared with other therapeutic options.

Identification of COL1A1:PDGFB as the fusion genes involved in the $t(17 ; 22)$ present in more than $90 \%$ of patients with DFSP has initiated investigation of the PDGFR-inhibitor imatinib. Imatinib is small-molecule inhibitor of PDGFR and several other tyrosine kinases, including $\mathrm{ABL}, \mathrm{KIT}, \mathrm{ARG}$, and
FMS. ${ }^{35}$ Imatinib has been investigated in patients with metastatic DFSP and those with localized DFSP not suitable for definitive surgery. Patients with metastatic disease who took imatinib showed significant clinical activity, with more than $50 \%$ ( 5 of 7 published cases) experiencing objective responses. ${ }^{36-40}$ Those with locally advanced disease not suitable for surgical resection also had significant activity. McArthur et al. ${ }^{39}$ reported objective responses in 8 of 8 cases of DFSP with locally advanced disease. All patients became diseasefree either after experiencing complete responses to imatinib or undergoing surgical resection after experiencing partial responses to imatinib. Based on these data, several neoadjuvant studies are underway. Although patients taking imatinib have shown high rates of clinical activity in DFSP with $t(17 ; 22)$, those with DFSP-like tumors with high-grade morphology but lacking $t(17 ; 22)$ or with complex cytogenetics may not respond to imatinib. ${ }^{37,39}$ Only 2 of 4 patients experienced response to imatinib, and this was short in 1 patient. ${ }^{36,37,39}$ Therefore, in selected cases, especially those with high-grade morphology, routine pathology should be supported by molecular analyses of the tumor with cytogenetics, in situ fluorescence hybridization (FISH), or comparative genomic hybridization (CGH). Cytogenetics is widely available, and specialist centers in sarcoma pathology can perform FISH on formalin-fixed paraffin-embedded material.

Evidence is now emerging that inhibition of PDGFR with drugs such as imatinib is a useful therapeutic strategy for treating unresectable DFSP. Radiotherapy is also a treatment option; however, the impressive results of imatinib coupled with surgical excision of residual masses suggest that this approach is worthy of ongoing investigation and use in selected patients who are not primary surgical candidates.

\section{Dose and Duration of Targeted Therapy for DFSP}

The high rates of clinical activity of imatinib in treating DFSP warrant the questions of how much and how long. This has become an issue because some patients with initially unresectable disease experienced complete response with a strategy of treating until maximum response, which for some was after 6 months of therapy.$^{39}$ This approach is being investigated further. ${ }^{41}$ In contrast, other current studies have prospectively 
planned resection of the DFSP after a defined period..$^{42}$ Collective analyses of data from these studies may provide useful information about the optimal duration of therapy. However, treatment to maximum response before surgical excision of residual tumor, or resection of the tumor bed, will probably be a preferred approach given the potential to limit surgical morbidity and the lack of any practical concern about the development of distant metastases during targeted medical therapy.

Responses of DFSP to imatinib have been obtained at daily doses of $400 \mathrm{mg}, 600 \mathrm{mg}$, and $800 \mathrm{mg}$. However, because most experience has involved $800 \mathrm{mg}$, this dose is the one currently approved. Compared with $400 \mathrm{mg}$, a starting dose of $800 \mathrm{mg}$ of imatinib has been associated with higher rates of edema, fatigue, and gastrointestinal and hematologic toxicity. ${ }^{43}$ Patients with gastrointestinal stromal tumors whose doses were escalated to $800 \mathrm{mg}$ after progressing on an initial dose of $400 \mathrm{mg}$ experienced better toxicity than those whose imatinib therapy was initiated at $800 \mathrm{mg}$ daily. ${ }^{44}$ Therefore, one potential approach to minimize the toxicity of $800 \mathrm{mg}$ of imatinib is to begin at $400 \mathrm{mg}$ and escalate the dose in a stepwise manner. Further data are required to determine if this approach of gradual intrapatient dose-escalation has equivalent efficacy to beginning therapy at higher doses.

\section{Neoadjuvant and Adjuvant Therapy for DFSP}

Because the major cause of treatment failure in DFSP is local recurrence, a neoadjuvant approach of targeted therapy or radiotherapy in DFSP may prevent local occurrence and potentially limit surgical morbidity. This approach has gained support in the management of soft tissue sarcoma where neoadjuvant treatment with radiotherapy is an option in cases with significant risks for local recurrence. ${ }^{30}$ As discussed previously, experience with neoadjuvant radiotherapy for treating DFSP is limited. More experience has been reported for neoadjuvant imatinib in a highly selected patient population..$^{39}$ Formal prospective studies are underway, but preliminary results have been encouraging. ${ }^{41,42}$ Although routine neoadjuvant imatinib in patients with resectable disease currently cannot be supported, neoadjuvant targeted therapy with imatinib has a place in treating marginally resectable cases given the growing experience in patients with locally advanced disease. Ideally, this application of imatinib should be part of a clinical trial to gather more data in this uncommon disease.

Patients at high risk for local recurrence, such as those with fibrosarcomatous histology, positive margins, or high mitotic count, are obvious candidates for effective adjuvant therapies. Among these risk factors, positive margins have been associated with a very high risk for further recurrence. ${ }^{2}$ Therefore, the goal of surgery is to obtain clear margins; adjuvant therapies are not substitutes for inadequate surgery. Nonetheless, some patients with positive margins are not candidates for further surgical excision because of the associated functional or cosmetic morbidity, and therefore adjuvant therapies can be considered.

Several studies have reported good outcomes for patients with positive margins treated with adjuvant radiotherapy after surgery. $33,34,45,46$ Although these studies report limited follow-up compared with larger surgical series, radiotherapy is an option for patients who cannot undergo further surgery and have positive margins or other risk factors, such as fibrosarcomatous histology or high mitotic count. Currently, no data are available on the use of imatinib in the adjuvant setting. Given the difficulty in performing randomized trials in an uncommon disease with such good outcomes with surgery alone, data will probably not become available to guide clinicians on the adjuvant use of PDGFR inhibitors in DFSP. Therefore, the adjuvant use of imatinib currently cannot be supported, particularly because the management of local recurrence with surgery, surgery plus radiotherapy, or imatinib followed by surgery has shown such impressive results.

\section{Conclusions and Current Indications for Targeted Therapy}

Because of the excellent rates of disease control obtained with aggressive surgical approaches, surgery continues to be the focus of optimal management of DFSP. Adjuvant radiotherapy may also offer some benefit to selected patients. Recent data have shown substantial activity of the targeted therapy imatinib in patients with unresectable disease, leading to approval by the FDA and European Agency for the Evaluation of Medicinal Products for this indication. High rates of clinical activity of imatinib in DFSP further reinforce the value of targeting specific molecular abnormalities in human malignancy. 
Molecular Targeting of Dermatofibrosarcoma Protuberans

\section{References}

1. U.S. Food and Drug Administration. FDA approves imatinib mesylate (Gleevec) as a single agent for the treatment of multiple indications. Available at: http://www.fda.gov/cder/offices/OODP/ whatsnew/imatinib200610.htm. Accessed April 9, 2007.

2. Bowne WB, Antonescu CR, Leung DH, et al. Dermatofibrosarcoma protuberans: a clinicopathologic analysis of patients treated and followed at a single institution. Cancer 2000;88:2711-2720.

3. Fiore M, Miceli R, Mussi C, et al. Dermatofibrosarcoma protuberans treated at a single institution: a surgical disease with a high cure rate. J Clin Oncol 2005;23:7669-7675.

4. Chang CK, Jacobs IA, Salti GI. Outcomes of surgery for dermatofibrosarcoma protuberans. Eur J Surg Oncol 2004;30:341-345.

5. Hsi ED, Nickoloff BJ. Dermatofibroma and dermatofibrosarcoma protuberans: an immunohistochemical study reveals distinctive antigenic profiles. J Dermatol Sci 1996;11:1-9.

6. Kahn HJ, Fekete E, From L. Tenascin differentiates dermatofibroma from dermatofibrosarcoma protuberans: comparison with CD34 and factor XIIIa. Hum Pathol 2001;32:50-56.

7. Hsi BL, Xiao S, Fletcher JA. Chromogenic in situ hybridization and FISH in pathology. Methods Mol Biol 2002;204:343-351.

8. Haycox CL, Odland PB, Olbricht SM, Piepkorn M. Immunohistochemical characterization of dermatofibrosarcoma protuberans with practical applications for diagnosis and treatment. J Am Acad Dermatol 1997;37(3 Pt 1):438-444.

9. Cribier B, Noacco G, Peltre B, Grosshans E. Stromelysin 3 expression: a useful marker for the differential diagnosis dermatofibroma versus dermatofibrosarcoma protuberans. J Am Acad Dermatol 2002;46:408-413.

10. Mentzel T, Beham A, Katenkamp D, et al. Fibrosarcomatous ("highgrade") dermatofibrosarcoma protuberans: clinicopathologic and immunohistochemical study of a series of 41 cases with emphasis on prognostic significance. Am J Surg Pathol 1998;22:576-587.

11. Simon MP, Pedeutour F, Sirvent N, et al. Deregulation of the plateletderived growth factor B-chain gene via fusion with collagen gene COL1A1 in dermatofibrosarcoma protuberans and giant-cell fibroblastoma. Nat Genet 1997;15:95-98.

12. Sjoblom T, Shimizu A, O'Brien KP, et al. Growth inhibition of dermatofibrosarcoma protuberans tumors by the platelet-derived growth factor receptor antagonist STI571 through induction of apoptosis. Cancer Res 2001;61:5778-5783.

13. Shimizu A, O'Brien KP, Sjoblom $T$, et al. The dermatofibrosarcoma protuberans-associated collagen type Ialpha1/platelet-derived growth factor (PDGF) B-chain fusion gene generates a transforming protein that is processed to functional PDGF-BB. Cancer Res 1999; 59:3719-3723.

14. Sirvent N, Maire G, Pedeutour F. Genetics of dermatofibrosarcoma protuberans family of tumors: from ring chromosomes to tyrosine kinase inhibitor treatment. Genes Chromosomes Cancer 2003; 37:1-19.

15. Linn SC, West RB, Pollack JR, et al. Gene expression patterns and gene copy number changes in dermatofibrosarcoma protuberans. Am J Pathol 2003;163:2383-2395.

16. Gisselsson D, Hoglund M, O'Brien KP, et al. A case of dermatofibrosarcoma protuberans with a ring chromosome 5 and a rearranged chromosome 22 containing amplified COL1A1 and PDGFB sequences. Cancer Lett 1998;133:129-134.

17. Sonobe $\mathrm{H}$, Furihata $\mathrm{M}$, Iwata J, et al. Dermatofibrosarcoma protuberans harboring $\mathrm{t}(9 ; 22)(\mathrm{q} 32 ; \mathrm{q} 12.2)$. Cancer Genet Cytogenet 1999;110:14-18.
18. Sandberg AA, Bridge JA. Updates on the cytogenetics and molecular genetics of bone and soft tissue tumors. Dermatofibrosarcoma protuberans and giant cell fibroblastoma. Cancer Genet Cytogenet 2003;140:1-12.

19. Wang J, Hisaoka M, Shimajiri S, et al. Detection of COL1A1-PDGFB fusion transcripts in dermatofibrosarcoma protuberans by reverse transcription-polymerase chain reaction using archival formalinfixed, paraffin-embedded tissues. Diagn Mol Pathol 1999;8:113-119.

20. Parker TL, Zitelli JA. Surgical margins for excision of dermatofibrosarcoma protuberans. J Am Acad Dermatol 1995;32(2 Pt 1): 233-236.

21. Lindner NJ, Scarborough MT, Powell GJ, et al. Revision surgery in dermatofibrosarcoma protuberans of the trunk and extremities. Eur J Surg Oncol 1999;25:392-397.

22. Houghton AN, Bichakjian CK, Coit DG, et al. The NCCN Melanoma Guidelines, version 1, 2007. Available at: http://www.nccn. org/professionals/physician_gls/PDF/melanoma.pdf. Accessed March 18, 2007.

23. Miller SJ, Alam M, Andersen J, et al. The NCCN Basal Cell and Squamous Cell Skin Cancers Guidelines, version 1, 2007. Available at: http://www.nccn.org/professionals/physician_gls/default.asp. Accessed April 9, 2007.

24. Garcia C, Holman J, Poletti E. Mohs surgery: commentaries and controversies. Int J Dermatol 2005;44:893-905.

25. Gloster HM Jr, Harris KR, Roenigk RK. A comparison between Mohs micrographic surgery and wide surgical excision for the treatment of dermatofibrosarcoma protuberans. J Am Acad Dermatol 1996; 35:82-87.

26. Nouri K, Lodha R, Jimenez G, Robins P. Mohs micrographic surgery for dermatofibrosarcoma protuberans: University of Miami and NYU experience. Dermatol Surg 2002;28:1060-1064; discussion 1064.

27. Snow SN, Gordon EM, Larson PO, et al. Dermatofibrosarcoma protuberans: a report on 29 patients treated by Mohs micrographic surgery with long-term follow-up and review of the literature. Cancer 2004;101:28-38.

28. DuBay D, Cimmino V, Lowe $\mathrm{L}$, et al. Low recurrence rate after surgery for dermatofibrosarcoma protuberans: a multidisciplinary approach from a single institution. Cancer 2004;100:1008-1016.

29. Miller SJ, Alam M, Andersen J, et al. The NCCN Dermatofibrosarcoma Protuberans Guidelines, version 1, 2007. Available at: http://www.nccn.org/professionals/physician_gls/default.asp. Accessed April 9, 2006.

30. Demetri GD, Baker LH, Benjamin RS, et al. The NCCN Soft Tissue Sarcoma Guidelines, version 1, 2007. Available at: http://www.nccn. org/physician_gls/index.html.

31. Torreggiani WC, Al-Ismail K, Munk PL, et al. Dermatofibrosarcoma protuberans: MR imaging features. AJR Am J Roentgenol 2002; 178:989-993.

32. Thornton SL, Reid J, Papay FA, Vidimos AT. Childhood dermatofibrosarcoma protuberans: role of preoperative imaging. J Am Acad Dermatol 2005;53:76-83.

33. Suit H, Spiro I, Mankin HJ, et al. Radiation in management of patients with dermatofibrosarcoma protuberans. J Clin Oncol 1996; 14:2365-2369.

34. Ballo MT, Zagars GK, Pisters P, Pollack A. The role of radiation therapy in the management of dermatofibrosarcoma protuberans. Int J Radiat Oncol Biol Phys 1998;40:823-827.

35. Capdeville R, Buchdunger E, Zimmermann J, Matter A. Glivec (STI571, imatinib), a rationally developed, targeted anticancer drug. Nat Rev Drug Discov 2002;1:493-502. 
McArthur

36. Rubin BP, Schuetze SM, Eary JF, et al. Molecular targeting of plateletderived growth factor $\mathrm{B}$ by imatinib mesylate in a patient with metastatic dermatofibrosarcoma protuberans. J Clin Oncol 2002;20:3586-3591.

37. Maki RG, Awan RA, Dixon RH, et al. Differential sensitivity to imatinib of 2 patients with metastatic sarcoma arising from dermatofibrosarcoma protuberans. Int J Cancer 2002;100:623-626.

38. Mizutani $K$, Tamada $Y$, Hara $K$, et al. Imatinib mesylate inhibits the growth of metastatic lung lesions in a patient with dermatofibrosarcoma protuberans. Br J Dermatol 2004;151:235-237.

39. McArthur GA, Demetri GD, van Oosterom A, et al. Molecular and clinical analysis of locally advanced dermatofibrosarcoma protuberans treated with imatinib: Imatinib Target Exploration Consortium Study B2225. J Clin Oncol 2005;23:866-873.

40. Labropoulos SV, Fletcher JA, Oliveira AM, et al. Sustained complete remission of metastatic dermatofibrosarcoma protuberans with imatinib mesylate. Anticancer Drugs 2005;16:461-466.

41. Ugurel S, Utikal J, Mohr P, et al. Imatinib in locally advanced dermatofibrosarcoma protuberans (DFSP): a phase II trial of the
Dermatologic Cooperative Oncology Group (DeCOG). J Clin Oncol 2006;24(18 suppl):535s.

42. Kerob D, Porcher R, Verola O, et al. Imatinib mesylate as a preoperative therapy in dermatofibrosarcoma: preliminary results of a multicentric phase II study. J Clin Oncol 2006;24(18 suppl):532s.

43. Verweij J, Casali PG, Zalcberg J, et al. Progression-free survival in gastrointestinal stromal tumours with high-dose imatinib: randomised trial. Lancet 2004;364:1127-1134.

44. Zalcberg JR, Verweij J, Casali PG, et al. Outcome of patients with advanced gastro-intestinal stromal tumours crossing over to a daily imatinib dose of $800 \mathrm{mg}$ after progression on $400 \mathrm{mg}$. Eur J Cancer 2005;41:1751-1757.

45. Sun LM, Wang CJ, Huang CC, et al. Dermatofibrosarcoma protuberans: treatment results of 35 cases. Radiother Oncol 2000; 57:175-181.

46. Dagan R, Morris CG, Zlotecki RA, et al. Radiotherapy in the treatment of dermatofibrosarcoma protuberans. Am J Clin Oncol 2005; 28:537-539. 